\title{
Variational Monte Carlo Studies of Pairing Symmetry for the $t-J$ Model on a Triangular Lattice
}

\author{
Tsutomu Watanabe ${ }^{1,2}$, Hisatoshi Yokoyama ${ }^{3}$, Yukio TAnakA ${ }^{1,2}$, Jun-ichiro Inoue $^{1}$ and \\ Masao OGATA ${ }^{4}$ \\ ${ }^{1}$ Department of Applied Physics, \\ Nagoya University, Nagoya 464-8603 \\ ${ }^{2}$ CREST Japan Science and Technology Corporation (JST) \\ ${ }^{3}$ Department of Physics, \\ Tohoku University, Sendai 980-8578 \\ ${ }^{4}$ Department of Physics, University of Tokyo, \\ Bunkyo-ku, Tokyo 113-0033
}

(Dated: March 23, 2018)

\begin{abstract}
As a model of a novel superconductor $\mathrm{Na}_{x} \mathrm{CoO}_{2} \cdot y \mathrm{H}_{2} \mathrm{O}$, a single-band $t$ - $J$ model on a triangular lattice is studied, using a variational Monte Carlo method. We calculate the energies of various superconducting (SC) states, changing the doping rate $\delta$ and sign of $t$ for small $J /|t|$. Symmetries of $s, d$, and $d+i d(p+i p$ and $f$ ) waves are taken up as candidates for singlet (triplet) pairing. In addition, the possibility of Nagaoka ferromagnetism and inhomogeneous phases is considered. It is revealed that, among the SC states, the $d+i d$ wave always has the lowest energy, which result supports previous mean-field studies. There is no possibility of triplet pairing, although the $f$-wave state becomes stable against a normal state in a special case $(\delta=0.5$ and $t<0)$. For $t<0$, the complete ferromagnetic state is dominant in a wide range of $\delta$ and $J /|t|$, which covers the realistic parameter region of superconductivity.
\end{abstract}

\section{INTRODUCTION}

The recent discovery of superconductivity [1] in $\mathrm{Na}_{0.35} \mathrm{CoO}_{2} \cdot 1.3 \mathrm{H}_{2} \mathrm{O}$ has aroused an active interest of solid state physicists, since this compound is regarded as a strongly correlated superconductor on a triangular lattice. For such magnetically frustrated lattices, novel superconducting (SC) features are expected, as compared to the celebrated cuprates on bipartite lattices. In particular, it is primarily important to determine the symmetry of its pairing potential. So far, there have been a number of experimental efforts trying to fix the pairing symmetry, e.g. measurements of nuclear spin lattice relaxation rate $T_{1}$ 2, 3, 4], Knight Shift [2, 5] by NMR, and by $\mu \mathrm{SR}$ 6, 7]. At present, however, the results of these researches are not necessarily consistent to each another; the pairing symmetry has not yet been established.

As for theory, although some studies regard multiband effects as important, [8, 9, 10] many treat this issue with reduced single-band models on a triangular lattice. The single-band studies are roughly classified into two categories, namely ones applying weakcoupling techniques to Hubbard-type models, like perturbation expansions [11], a random-phase approximation 12 and a fluctuation exchange approximation (FLEX) 13. and ones starting with $t$ - $J$-type models in the strong-coupling limit. 14, 15, 16, 17, 18] Although the weak-coupling studies took mutually different approaches for different models, they arrived at a conclusion that $f$-wave spin-triplet symmetries are competitive with or even dominant over $d$-wave spin-singlet symmetries. On the other hand for $t$ - $J$-type models, it is unanimously shown that $d_{x^{2}-y^{2}}+i d_{x y}$-wave symmetries are widely sta- ble against other symmetries as well as metallic states, using slave-boson mean-field approximations 14, 15, 16], which barely respect the local constraint of no double occupation, and a Gutzwiller approximation [17], which takes account of the constraint on an average. Thus, the mean-field results of the strong-coupling theories disagree with the results of the weak-coupling ones, in contrast with the case of a square lattice, where the conclusion of the $d_{x^{2}-y^{2}}$ wave has been drawn in both theories.

In this paper, we study the $t$ - $J$ model on a triangular lattice, using a variational Monte Carlo (VMC) method, which exactly treats the local constraint of no double occupation. Our main purposes are (i) to determine the pairing symmetry of superconductivity within the $t-J$ model with this more accurate method, and (ii) to check the possibility of spin-triplet superconductivity in this strong-coupling model. Besides the SC states, (iii) we consider the stability of Nagaoka ferromagnetism [19, 20, 21, 22] and inhomogeneous phases. Based on these calculations, we argue the propriety of modeling for cobaltates.

The organization of this paper is as follows: In $\S 2$, the formulation used is described. Sections 3 and 4 are assigned to the results and discussions on the stability of the singlet and triplet SC states, respectively. In $\S 5$, we treat ferromagnetism and phase separation. In $\S 6$, we recapitulate our results and address the relevance to cobaltates. A part of the present results has been reported. 18] 
(a)
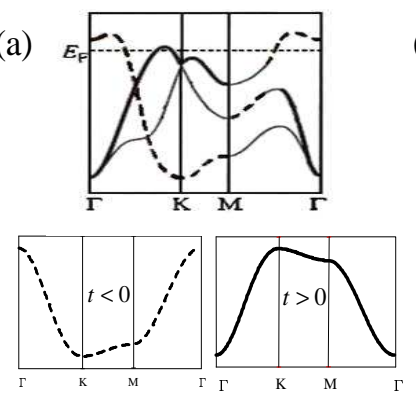

(b)

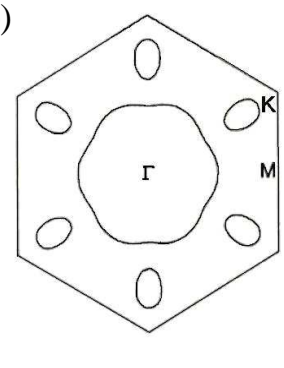

FIG. 1: (a) Upper panel: Schematic plot of the band structure for $\mathrm{Na}_{0.5} \mathrm{CoO}_{2}$, following an LDA calculation 24]. Lower panels: Dispersion of single-band tight binding models on a triangular lattice for $t<0$ and $t>0$. (b) Hole Fermi surfaces of the $\mathrm{CoO}$ planes in $\mathrm{Na}_{0.5} \mathrm{CoO}_{2}$, due to the same LDA.

\section{FORMULATION}

Generally, the $3 d$ orbitals are split into $t_{2 g}$ and $e_{g}$ orbitals by an octahedral crystal field. In this material, the $t_{2 g}$ orbitals of $\mathrm{Co}$ are further split into the levels of $a_{1 g} \quad\left[=\left(d_{x y}+d_{y z}+d_{z x}\right) / \sqrt{3}\right]$ and $e_{g}^{\prime}$ $\left[=\left(d_{x y}+e^{ \pm i \frac{2 \pi}{3}} d_{y z}+e^{ \pm i \frac{4 \pi}{3}} d_{z x}\right) / \sqrt{3}\right][23]$, by a shift of oxygen vertical to the $\mathrm{CoO}_{2}$ layers. Due to an LDA calculation for $\mathrm{Na}_{0.5} \mathrm{CoO}_{2}[24]$, the bands near the Fermi surface [Fig. 1(b)] are involved in a Co $t_{2 g}$ manifold [Fig. 1(a)]. Since $\mathrm{Na}_{0.35} \mathrm{CoO}_{2} \cdot 1.3 \mathrm{H}_{2} \mathrm{O}$ has the same structure as $\mathrm{Na}_{0.5} \mathrm{CoO}_{2}$, except for the intercalation of water molecules and the sodium concentration, one may assume the conduction bands for $\mathrm{Na}_{0.35} \mathrm{CoO}_{2} \cdot 1.3 \mathrm{H}_{2} \mathrm{O}$ are similarly formed from the $t_{2 g}$ manifold. The band which forms the large Fermi surface around the $\Gamma$ point (Fig. 1(b)) has dominant $a_{1 g}$ character, whereas the band which forms the small pocket Fermi surfaces on the $\Gamma-\mathrm{K}$ lines have mixed $a_{1 g}$ and $e_{g}^{\prime}$ character. Thus, we call the band indicated by dashed (thick solid) lines in Fig. I(a) the $a_{1 g}\left(e_{g}^{\prime}\right)$ band. Many previous studies have assumed that $\mathrm{Na}_{0.35} \mathrm{CoO}_{2} \cdot 1.3 \mathrm{H}_{2} \mathrm{O}$ is basically described by singleband models of the $a_{1 g}$ or $e_{g}^{\prime}$ band, as mentioned.

Along this line, we consider a single-band $t$ - $J$ model on an extended square lattice,

$$
\begin{aligned}
\mathcal{H}=\mathcal{H}_{t}+\mathcal{H}_{J}=- & \sum_{(i, j) \sigma} t_{i j} \mathcal{P}_{\mathrm{G}}\left(c_{i \sigma}^{\dagger} c_{j \sigma}+\text { H.c. }\right) \mathcal{P}_{\mathrm{G}} \\
& +\sum_{(i, j)} J_{i j}\left(\mathbf{S}_{i} \cdot \mathbf{S}_{j}-\frac{1}{4} n_{i} n_{j}\right),
\end{aligned}
$$

where $\mathcal{P}_{\mathrm{G}}=\prod_{i}\left(1-n_{i \uparrow} n_{i \downarrow}\right)$. The sum of site pairs $(i, j)$ is restricted as follows: $t_{i j}=t$ and $J_{i j}=J(>0)$ for the nearest-neighbor pairs, $t_{i j}=t^{\prime}$ and $J_{i j}=J^{\prime}(>0)$ for the next-nearest-neighbor pairs only in one diagonal direction, and $t_{i j}=J_{i j}=0$ otherwise (see Fig. 2). Except for the case of $t<0$ and $\delta \sim 0.5(\S 4)$, where the degen-

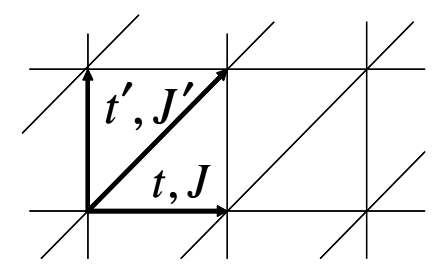

FIG. 2: Lattice structure of the model used in this study, with its parameters.

eracy at $\varepsilon=\varepsilon_{\mathrm{F}}$ is serious, we put $t^{\prime}=t$ and $J^{\prime}=J$. Here, $\delta$ indicates the hole concentration: $\delta=|1-n|$ with $n=N_{\mathrm{e}} / N_{\mathrm{s}}\left(N_{\mathrm{e}}\right.$ : electron number, $N_{\mathrm{s}}$ : site number $)$ - This parameter setting makes no difference with the isotropic triangular lattice.

Let us make a remark on the sign of $t$. In the lower panels of Fig. 1(a), the tight-binding bands of the present model,

$$
\varepsilon_{\mathbf{k}}=-2 t\left(\cos k_{x}+\cos k_{y}\right)-2 t^{\prime} \cos \left(k_{x}+k_{y}\right),
$$

with $t^{\prime}=t$ are depicted for $t>0$ and $t<0$. Compared with the LDA band structure (upper panel of Fig. 1(a)), it seems appropriate that the band eq. (2) with $t<0$ $[t>0]$ should be connected to the $a_{1 g}$ (dashed lines) $\left[e_{g}^{\prime}\right.$ (bold lines)] band. In $\mathrm{Na}_{x} \mathrm{CoO}_{2} \cdot y \mathrm{H}_{2} \mathrm{O}$, the electron density is larger than 1, i.e. electron-doped case. To treat electron-doped cases in the framework of $t$ - $J$-type models, namely in the electron density of $0 \leq n \leq 1$ without double occupation, we take advantage of a particle-hole transformation. Thereby, an electron-doped case with $t<0$, which corresponds to the $a_{1 g}$ band (lower left panel of Fig. [(a)), is mapped to a hole-doped case with $t>0$ in $t-J$ model of eq. (1). We use this sign convention throughout this paper. In fact, the $t<0$ band, which corresponds to the $e_{g}^{\prime}$ band, has a van Hove singularity at $\delta=0.5$, as the $e_{g}^{\prime}$ band does near the $\mathrm{K}$ points. So far, the majority of theoretical studies 11, 12, 15, 16, 17, 25 have considered that the large Fermi surface around the $\Gamma$ point mainly contributes, whereas some papers have regarded the isolated pocket Fermi surfaces or the van Hove singularities as important for superconductivity. [9, 13, 14] In this paper, we consider both cases, $t<0$ and $t>0$.

Since the cobalt atoms in the present compound are connected through edge-shared oxygen cages, the effective value of $J /|t|$ is expected to be fairly small. Accordingly, we principally discuss the region of small $J /|t|$ in this paper.

We move on to the method used. To consider strongly correlated systems, more accurate treatment of the local constraint is necessary beyond the mean field level. For this purpose, a variational Monte Carlo (VMC) method [26, 27, 28] is appropriate. In this method, variational expectation values of many-body wave functions are numerically estimated with a Monte Carlo procedure, in which the local constraint, e.g. $\mathcal{P}_{\mathrm{G}}$ in eq. (1), is exactly 
TABLE I: $\Delta_{\mathbf{k}}$ for various pairing symmetries.

\begin{tabular}{c|c}
\hline Symmetry & $\Delta_{\mathbf{k}}$ \\
\hline$s$ & $\Delta$ \\
ext. $s(1)$ & $\Delta\left(\cos k_{x}+\cos k_{y}\right)$ \\
ext. $s(2)$ & $\Delta\left[\cos k_{x}+\cos \left(k_{x}+k_{y}\right)+\cos k_{y}\right]$ \\
$d$ & $\Delta\left(\cos k_{x}-\cos k_{y}\right)$ \\
$d+i d$ & $\Delta\left[\cos k_{x}+e^{i \frac{2 \pi}{3}} \cos \left(k_{x}+k_{y}\right)+e^{i \frac{4 \pi}{3}} \cos k_{y}\right]$ \\
$p+i p$ & $\Delta\left[\sin k_{x}+e^{i \frac{2 \pi}{3}} \sin \left(k_{x}+k_{y}\right)+e^{i \frac{4 \pi}{3}} \sin k_{y}\right]$ \\
$f$ & $\Delta\left[\sin k_{x}-\sin \left(k_{x}+k_{y}\right)+\sin k_{y}\right]$ \\
\hline
\end{tabular}

satisfied. Actually, it has been shown that the VMC method is effective for a variety of analyses of the $t$ - Jtype models on square lattices without (or with small) frustration [29, 30]. At least, we believe this method is reliable to discuss the mutual stability among various pairing symmetries. In this paper, we apply this method to the model eq. (11) to study the stability of various SC states.

For $t$-J-type models, a Gutzwiller-type variational wave function is a good starting point, 29, 30, 31] unlike Hubbard-type models. 32 For the normal state, we use the original Gutzwiller wave function $\Psi_{\mathrm{n}}=\mathcal{P}_{\mathrm{G}} \Phi_{\mathrm{F}}$ $\left(\Phi_{\mathrm{F}}\right.$ : Fermi sea). 33] A SC state with a fixed electron number 34] is given as,

$$
\left|\Psi_{\mathrm{sc}}\right\rangle=\mathcal{P}_{\mathrm{G}}|\mathrm{BCS}\rangle=\mathcal{P}_{\mathrm{G}}\left(\sum_{\mathbf{k}} \varphi_{\mathbf{k}} c_{\mathbf{k} \uparrow}^{\dagger} c_{-\mathbf{k} \downarrow}^{\dagger}\right)^{\frac{N_{\mathrm{e}}}{2}}|0\rangle,
$$

with

$$
\varphi_{\mathbf{k}}=\frac{u_{\mathbf{k}}}{v_{\mathbf{k}}}=\frac{\Delta_{\mathbf{k}}}{\varepsilon_{\mathbf{k}}+\sqrt{\varepsilon_{\mathbf{k}}^{2}+\Delta_{\mathbf{k}}^{2}}} .
$$

Anisotropy of the pairing symmetry is introduced in $\Delta_{\mathbf{k}}$. We consider $s$, extended- $s$ (two kinds), $d$ and $d+i d$ waves as candidates for singlet pairing, and $p+i p$ and $f$ waves for triplet pairing. They are summarized in Table I. Symmetries of $s, p+i p$ and $d+i d$ waves have no line node in $\Delta_{\mathbf{k}}$, whereas $d$ and $f$ waves have line nodes. The timereversal symmetry is broken for $d+i d$ and $p+i p$ waves. In the present calculation, $\Delta$ is the only variational parameter, which is connected to the SC gap, in particular for a high-doping region, but is not the gap itself in contrast with weak-coupling theories. Except for half filling, we may consider $\Psi_{\text {sc }}$ with finite $\Delta$ is a SC state, while at half filling $\Psi_{\mathrm{sc}}$ is an insulating RVB state. 35] We fix $\mu$ at $\mu_{0}$, the chemical potential for the noninteracting system, because $\mu$ dependence of $E_{\text {tot }}$ is small, and $\mu$ does not seem an essential variable in a fixed- $N_{\mathrm{e}}$ formulation.

Since the number of variational parameters is at most one in this study, an usual VMC procedure [28, 34] sufficiently works. To obtain an accurate energy difference (condensation energy), we have taken a sufficient number of samples to reduce the statistical errors, and kept the sampling interval long enough to ensure statistical independence of the samples. Here, we collect samples as many as $10^{5}-10^{7}$, which yield the error in energy of approximately $10^{-4}|t|$. We use the systems of $N_{s}=L \times L$ ( $L=4-18)$ with the periodic-antiperiodic boundary conditions, and the electron densities satisfying the closed shell condition.

\section{STABILITY OF SINGLET PAIRINGS}

In this section, we discuss the symmetries of singlet pairing, $s$, extended- $s, d$ and $d+i d$ waves.

To begin with, we consider $s$-type pairings. Figure 3 shows the hopping energy $E_{t}=\left\langle\mathcal{H}_{t}\right\rangle$ and exchange energy $E_{J}=\left\langle\mathcal{H}_{J}\right\rangle$ for the homogeneous $s$-wave symmetry, as a function of $\Delta$. For both $t<0$ and $t>0$, each of $E_{t} /|t|$ and $E_{J} / J$ is a monotonically increasing function of $\Delta$, indicating the energy minimum is at $\Delta=0$ for any value of $J / t$; the $s$-wave state is not stable against the normal state. We also confirmed for various values of $\delta$ that this situation does not change. This result is natural for the homogeneous $s$ wave, which favors onsite pairing. As for the two kinds of extended- $s$ waves, types (1) and (2), although $E_{t} /|t|$ is a rapidly increasing function of $\Delta, E_{J} / J$ has a shallow minimum at a finite value of $\Delta$. This means these symmetries become stable for finite values of $J / t$, unlike the homogeneous $s$ wave. We confirmed, however, that the energy minimum is at $\Delta=0$ for a small value of $J /|t| \cdot[\underline{36}]$ We thus conclude that there is no possibility of the $s$ and extended- $s$ waves realized in the regime of our concern. We will not refer to these states, henceforth.

Next, we turn to the $d$ - and $d+i d$-wave pairings. Figures 4 and 5 show $E_{t} /|t|$ and $E_{J} / J$, respectively, of the $d+i d$ wave as a function of $\Delta /|t|$. In this case, $E_{t} /|t|$ is again a monotonically increasing function of $\Delta$, whereas $E_{J} / J$ conspicuously decreases and has a minimum at considerably large values of $\Delta$. Thereby, the $d+i d$ state becomes stable even for considerably small value of $J /|t|$ (e.g. $J / t \gtrsim 0.05$ for $\delta=0.04$ and $t>0$ ). Similar behaviors of $E_{t}$ and $E_{J}$ can be seen for the $d$-wave state, as seen in Figs. [6 and 7] From these figures, we find that the $d+i d$ and $d$ waves have similar energies. To see actual energy reduction, we plot in Fig. 8 the total energy $E_{\text {tot }}$ $\left(=E_{t}+E_{J}\right)$ for $J /|t|=0.3$ and $\delta=0.04$ and 0.12 , as an example. For both $d$ and $d+i d$ waves, $E_{\text {tot }}$ has a clear minimum at large values of $\Delta$ for both signs of $t$; the SC states of $d$ and $d+i d$ waves are appreciably stable near half filling. For $\delta \sim 0$, the minimum of the $d+i d$ wave is slightly lower than that of the $d$ wave. 38.

To consider the $\delta$ dependence of energy, we define a condensation energy, $E_{\mathrm{c}}$, as the difference of $E_{\text {tot }}$ between the minimized superconducting state and the normal state,

$$
E_{\mathrm{c}}=E\left(\Delta_{\min }\right)-E(0) .
$$

For $E_{\mathrm{c}}<0$, the superconducting state is stable against the normal state. Plotted in Fig. 9 is $E_{\mathrm{c}}$ for the $d$ and $d+i d$ waves; $\left|E_{\mathrm{c}}\right|$ is a rapidly decreasing function of $\delta$. 

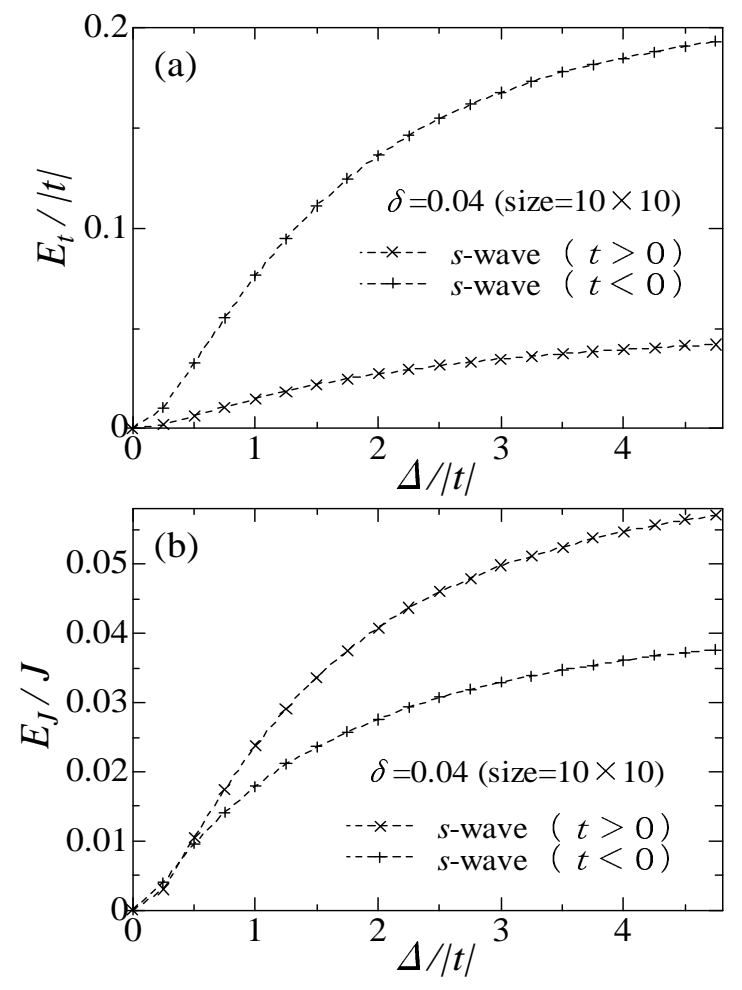

FIG. 3: (a) Hopping energy and (b) exchange energy of the homogeneous $s$-wave state as a function of $\Delta /|t|$ for $\delta=0.04$. The two cases, $t>0$ and $t<0$, are simultaneously shown. We take the origin of each energy as the value for $\Delta=0$, namely the normal state, $E_{t}(0)$ and $E_{J}(0)$; similar metrics are used in some figures following. The system used has $10 \times 10$ sites.

Near half filling, the $d+i d$-wave state is more stable than the $d$-wave state for both signs of $t$, probably because the symmetry of $\Delta_{k}$ of the former is more favorable to the band symmetry, which is not far from isotropic. The difference of $E_{\mathrm{c}}$, however, again becomes indistinguishable, as $\delta$ further increases, and $\left|E_{\mathrm{c}}\right|$ itself becomes small.

Note that $E_{\mathrm{c}}$ of the $d$ and $d+i d$ waves remains finite up to $\delta=0.24$ for $t>0$, and $\delta=0.60$ for $t<0$. Namely, the range of $\delta$ where the $d+i d$-wave state is dominant is much wider for $t<0$ than for $t>0$. This behavior agrees with the Gutzwiller approximation 17 even quantitatively. This difference is understood as follows: For $t<0$, the density of states $\rho(\varepsilon)$ increases as $\delta$ increases, and has a van Hove singularity at $\delta=0.5$, while, for $t>0, \rho(\varepsilon)$ is a monotonically decreasing function of $\delta$ until the band bottom. This difference yields different behaviors particularly in $E_{J}$, as seen in Figs. [5 and 7 For $t>0$, the minimum in $E_{J}$ smoothly increases as $\delta$ increases for both $d$ and $d+i d$ waves. On the other hand, for $t<0$, the minimum in $E_{J}$ once decreases as $\delta$ increase, and has the lowest value at $\delta \sim 0.36$ for the $d+i d$ wave [Fig. [5) (b)]; for the $d$ wave, the minimum in $E_{J}$ is stationary for $0.04 \lesssim \delta \lesssim 0.20$ [Fig. [7(b)]. For both waves, the decrease in $E_{J}$ is still conspicuous for $\delta$ as large as
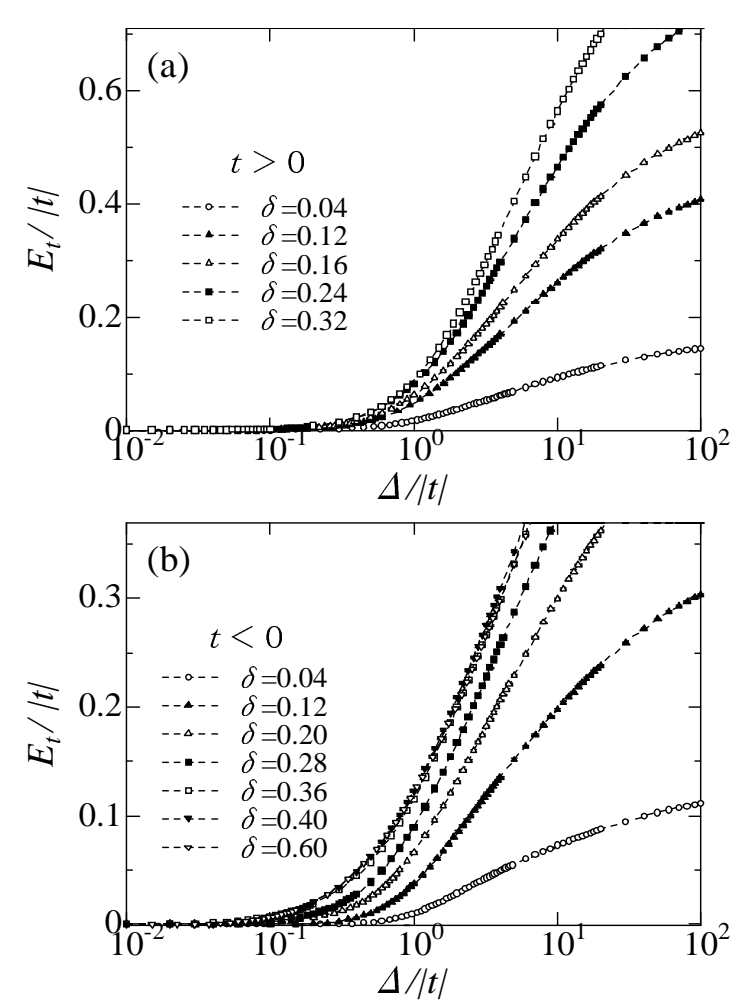

FIG. 4: Hopping energy of the $d+i d$-wave symmetry for several values of the hole density $\delta$. (a) $t>0$ and (b) $t<0$. The system size is $10 \times 10$. Statistical errors are smaller than the symbol sizes.

0.6. For this reason, the stability of the SC is enhanced particularly near the van Hove singularity point; we will return to this point in IIV

The above VMC results that the $d+i d$ wave is dominant qualitatively support the conclusions derived from mean-field approximations 14, 15, 16] and a Gutzwiller approximation. 17 For comparison, we plot in Fig 10 $E_{\text {tot }}\left(\Delta_{\text {min }}\right)$ of the $d+i d$-wave state by the present calculations and that of the Gutzwiller approximation. Although $E_{\text {tot }}\left(\Delta_{\min }\right)$ by VMC is somewhat lower than that by Gutzwiller approximation, the two curves exhibit similar doping dependence.

\section{POSSIBILITY OF TRIPLET PAIRINGS}

Ferromagnetic fluctuation, favored by triplet superconductivity, has been predicted by an LDA calculation, 39. and actually observed in an NMR- $T_{1}$ measurement for a superconducting sample. [4] In addition, many theories in the weak-coupling region have made conclusions of probable triplet superconductivity. In view of these suggestions, we study the stability of triplet symmetries, $p+i p$ and $f$ waves, in this section.

For triplet symmetries, the same VMC procedure is carried out in the $S^{z}=0$ sector. Having calculated ener- 

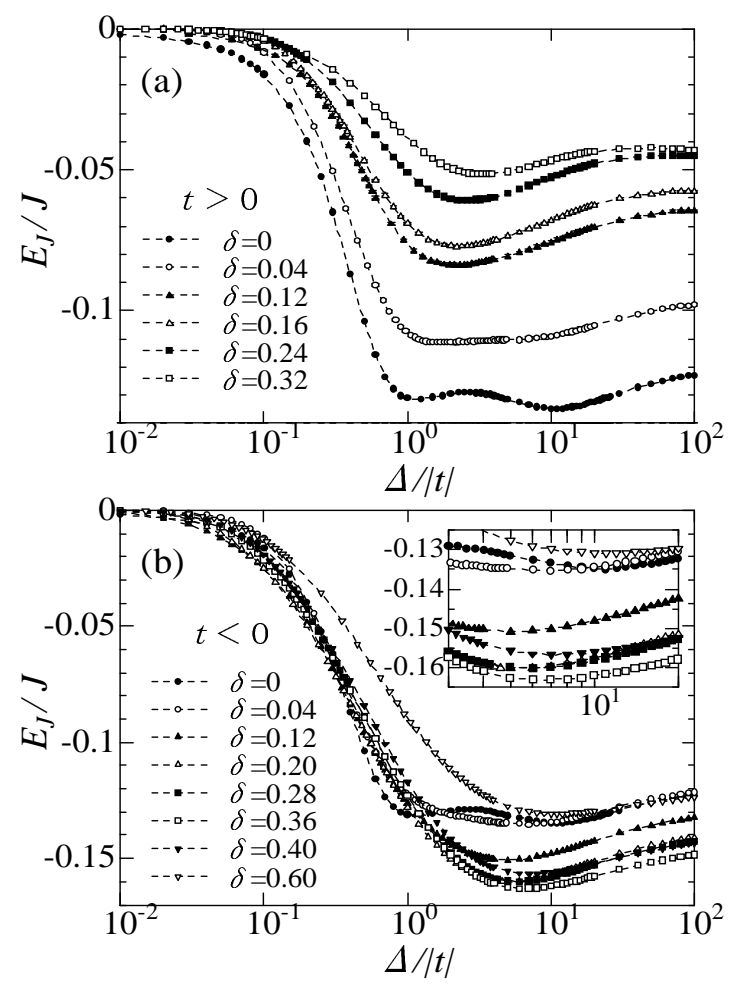

FIG. 5: Exchange energy of the $d+i d$-wave symmetry for several values of the hole density $\delta$. (a) $t>0$ and (b) $t<0$. The inset in (b) is the magnification of the minimum area. The system is the same as Fig 4

gies for various parameter values, it is found that $E_{J}$ is always a monotonically increasing function of $\Delta$. This is because the exchange term $\mathcal{H}_{J} / J$ with $J>0$ in eq. (10) is obviously unfavorable to a triplet state. Moreover, $E_{t} /|t|$ is also a monotonically increasing functions of $\Delta$, except for the case of $\delta \sim 0.5$ and $t<0$. Namely, the triplet superconducting states are always unstable against the normal state, excluding this special condition. In the following, therefore, we concentrate on this special case, $t<0$ and $\delta=0.5$, at which the Fermi energy is situated just at the van Hove singularity point.

At this point, severe level degeneracy arises due to the divergence of $\rho\left(\varepsilon_{\mathrm{F}}\right)$. As a result, for finite systems, the electron densities where the closed-shell condition is satisfied disappear near $\delta=0.5$. For example, this range is $0.4<\delta<0.6$ for $L=10$, as seen in Fig 9(b). To avoid this difficulty, we slightly change the value of $t^{\prime} / t$ from 1 to e.g. 0.999999, which lifts the degeneracy and make it possible to study $\delta=0.5$ without breaking the closed-shell condition. 40]

In Fig. 11(b), $E_{J}$ is shown for four symmetries. As mentioned above, $\mathcal{H}_{J}$ works disadvantageously to the triplet pairing symmetries; $E_{J}$ for the $p+i p$ and $f$ waves is again a monotonically increasing function of $\Delta$, in contrast with the behavior of the singlet pairing symmetries. Thus, the triplet SC states are never stabilized when $J /|t|$
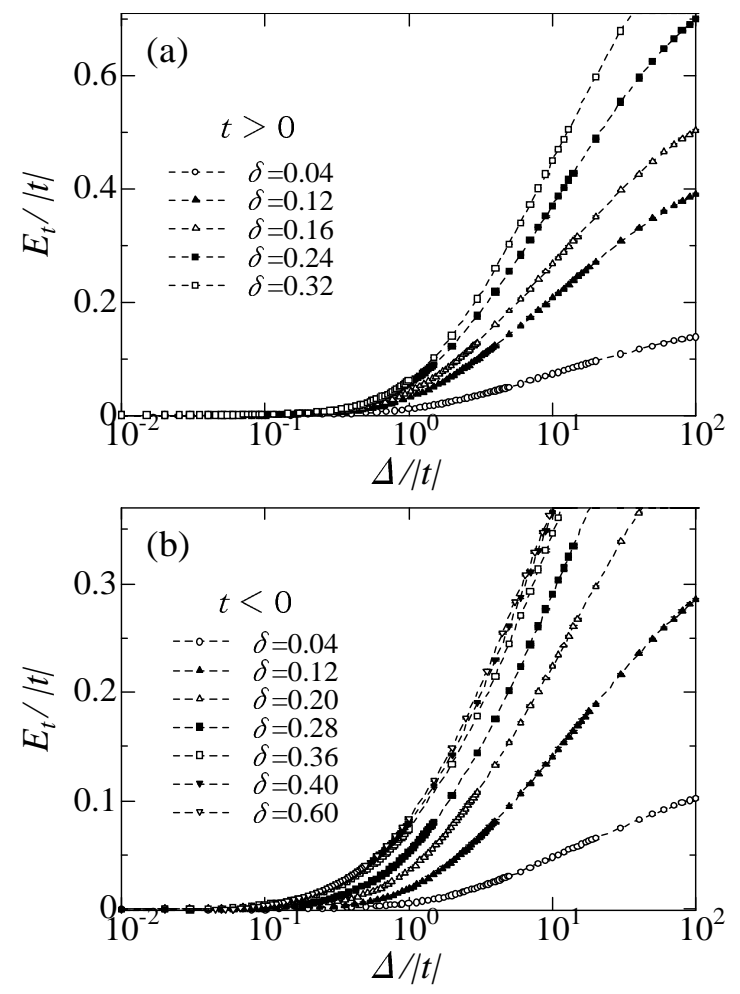

FIG. 6: Hopping energy of the $d$-wave symmetry for several values of the hole density $\delta$. (a) $t>0$ and (b) $t<0$. The system size is $10 \times 10$.

increases. In Fig. 111(a), $E_{t}$ is depicted for the same symmetries. Note that $E_{t}$ for the $f$ and $p+i p$ waves (solid symbols) has a shallow minimum at a finite value of $\Delta$. From this result, we find that the $f$-wave state becomes stable against the normal state $(\Delta=0)$ for small values of $J /|t|(J /|t| \lesssim 0.18)$. Compared with the singlet pairing symmetries, however, the decrease in $E_{t}$ for the triplet pairings is much smaller than that for the $d+i d$ and $d$ waves. Moreover, the singlet SC states gain energies in $E_{J}$ for finite values of $J / t$. We add the data of the $d$ and $d+i d$ waves at this density $(J /|t|=0.3)$ to Fig. 9(b); we find that the van Hove singularity appreciably contributes to superconductivity. Nonetheless, we will see that even this $d$ wave is defeated by ferromagnetism for small $J /|t|$.

To summarize, triplet superconductivity is not realized in the triangular $t-J$ model.

\section{FERROMAGNETISM AND PHASE SEPARATION}

In this section, we discuss ferromagnetic states and inhomogeneous phases, which are inseparable from the $t-J$ model.

Let us begin with the Nagaoka ferromagnetism. 19] Since Nagaoka's theorem (in the limit of $J /|t|, \delta \rightarrow 0$ ) 

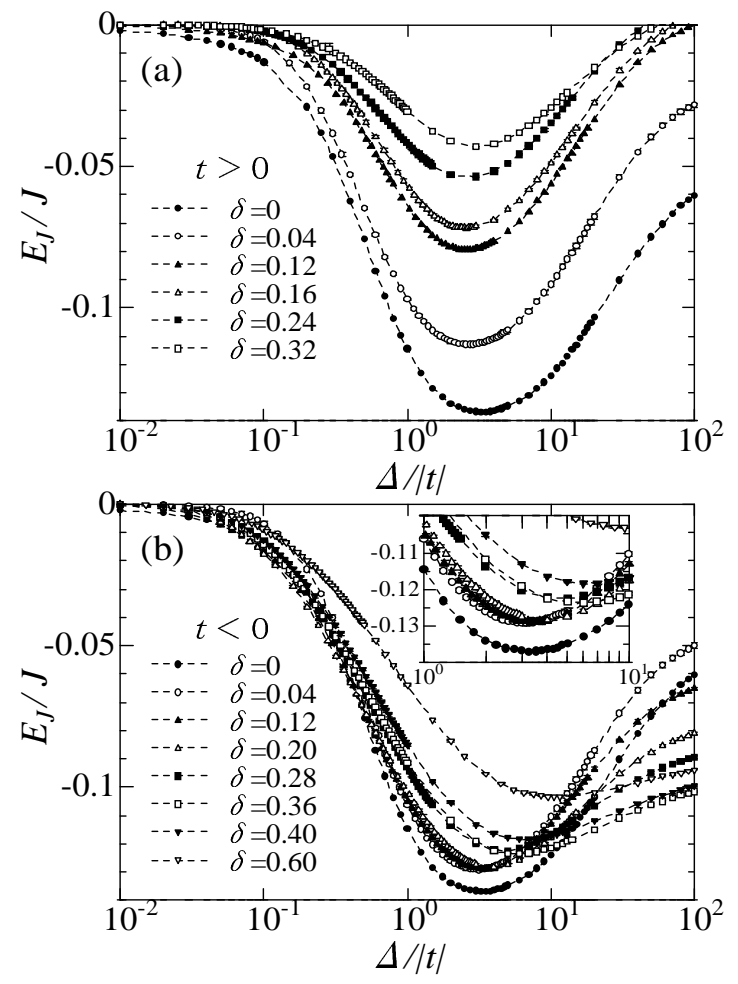

FIG. 7: Exchange energy of the $d$-wave symmetry for several values of the hole density $\delta$. (a) $t>0$ and (b) $t<0$. The inset in (b) is the magnification of the minimum area. The system is the same as Fig 6

holds for $t<0$ in a triangular lattice, a finite range of ferromagnetism is expected near this limit. Actually, it was shown using a variation scheme for the Hubbard model on a triangular lattice with $t<0$ that a Nagaoka itinerant ferromagnetism is widely stable. 20] For the $t$ - $J$ model with $t<0$, recent studies using a hightemperature expansion 21] showed the Nagaoka region to be $0<\delta \lesssim 0.85$ and $J / t \lesssim 0.5$, which is quite wider than that for a square lattice (possibly a partial ferromagnetism), namely, $0<\delta \lesssim 0.4$ and $J / t \lesssim 0.1$. 22, 29]

With these in mind, we look at our VMC results in the following. In Fig. 12] we show $E_{t}$ for the complete ferromagnetism (Nagaoka state), $E^{\mathrm{f}}$, and the $d+i d$-wave SC state, $E_{t}^{d+i d}$. For $t>0, E^{\mathrm{f}}$ is always higher than $E_{t}^{d+i d}$; thus the complete ferromagnetism never takes place, because $\left\langle\mathcal{H}_{J}\right\rangle=0$ for the Nagaoka state, while $E^{d+i d}$ further decreases due to the contribution of $\mathcal{H}_{J}$. Inversely, for $t<0, E^{\mathrm{f}}$ is appreciably smaller than $E_{t}^{d+i d}$ for most values of $\delta$, thereby there exist a region for small $J /|t|$ where the Nagaoka state is more stable than the $d+i d-$ wave state.

Comparing $E^{\mathrm{f}}$ and $E^{d+i d}$ for finite values of $J / t$, we determined the phase boundary, as shown in Fig. 131(b). The range of ferromagnetism is very broad, namely $\delta \leq$ 0.96 and $J /|t| \lesssim 0.7$, which is quantitatively consistent with a recent result of high-temperature expansion, 21]
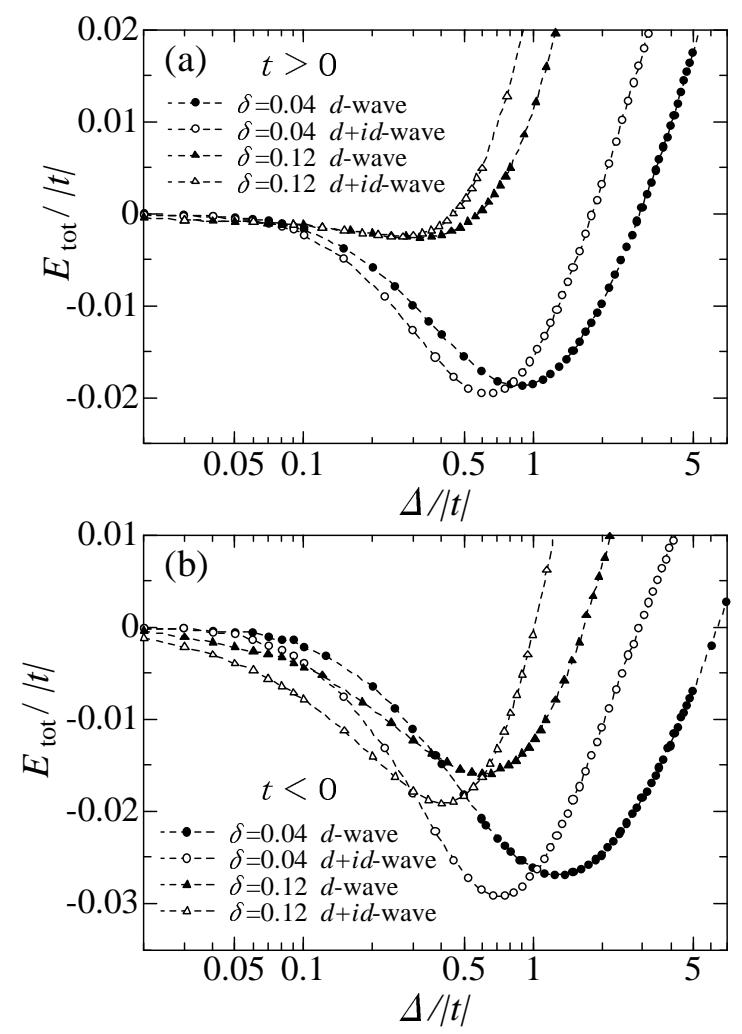

FIG. 8: Total energy of the $d$ - (solid symbols) and $d+i d$-wave (open symbols) states for $\delta=0.04$ and 0.12 . The system is of $10 \times 10$, and $J /|t|=0.3$. (a) $t>0$ and (b) $t<0$.

and is markedly wider than that for the square lattice estimated through the same VMC method. 22, 29] Consequently, for $t<0$, it is unlikely that superconductivity is realized in the region of small $J /|t|$ and intermediate $\delta$, even if we consider that the ferromagnetic region somewhat shrinks by improving the trial function of superconducting states.

Now, we turn to the phase separation in the triangular lattice. Since the exchange term in eq. (11) works as a kind of attractive potential, the $t-J$ model necessarily brings about a phase separation for large values of $J /|t|$. For the one-dimensional lattice, the boundary between homogeneous and inhomogeneous phases extends in the direction of large $J / t$ as $\delta$ decreases [4], whereas for the square lattice, the boundary approaches the origin $[(J / t, \delta)=(0,0)][29,42]$.

Here, we estimate the boundary in two distinct ways. (i) We suppose that the inhomogeneous state phase separates into the two hole densities, $\delta=0$ (half filling) and $\delta=1$ (empty), and the energy of this state is estimated as $E^{\mathrm{PS}}=(1-\delta) E^{\mathrm{AF}}$, where we adopt, as $E^{\mathrm{AF}}$, a precise value obtained by a quantum Monte Carlo method for the Heisenberg model, 43] in which the antiferromagnetic (AF) state with the $120^{\circ}$ structure is realized. The boundary is determined by comparing $E^{\mathrm{PS}}$ and $E^{d+i d}$. 

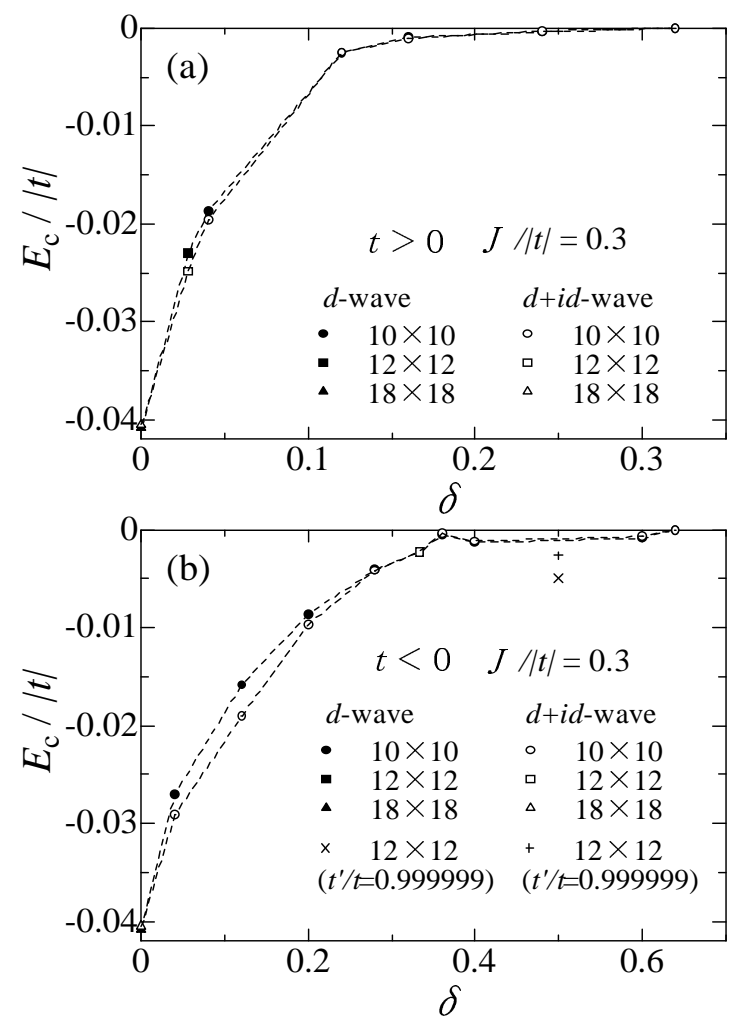

FIG. 9: Condensation energy of the $d$ - and $d+i d$-wave states as a function of doping rate $\delta$ for $J /|t|=0.3$. (a) $t<0$ and (b) $t>0$. In (b), the data for $\delta=0.5$ (the van Hove singularity point) is obtained under a different condition; for details, see IV Note that the range of abscissa is twice larger for $t<0$. The Data for $L=10,12$ and 18 are simultaneously plotted; system-size dependence is not severe.

Note that the reliability of this estimation increases as $\delta$ increases, but the boundary necessarily converges to $J /|t|=0$ as $\delta \rightarrow 0$. [44] (ii) Instead, we estimate the boundary near half filling, using the condition of intrinsic stability, $\partial^{2} E / \partial \delta^{2}>0$, for the $d+i d$-wave state. Since we actually substitute finite differences for $\partial^{2} E / \partial \delta^{2}$, the critical value of $J /|t|$ at half filling for $L \rightarrow \infty$ is likely to be smaller.

The results of (i) and (ii) are summarized in Fig. 13. For $t<0$, the critical value $J_{\mathrm{c}} /|t|$ is relatively small and barely depends on $\delta$ for large $\delta$. For $t>0, J_{\mathrm{c}} /|t|$ is fairly large for large $\delta$, and gradually decreases as $\delta$ decreases. For both signs of $t, J_{\mathrm{c}} /|t|$ abruptly decreases as $\delta$ approaches half filling; this tendency is analogous to the square lattice case. Although the possibility of phase separation still remains for a realistic value of $J /|t|$ near half filling, this is not the case with intermediate and large values of $\delta$.

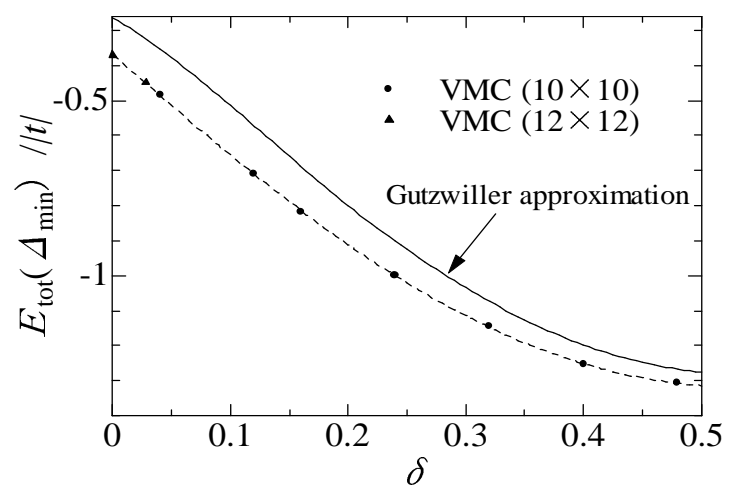

FIG. 10: Comparison of $E_{\text {tot }}\left(\Delta_{\text {min }}\right)$ of the $d+i d$-wave pairing between the present VMC and a Gutzwiller approximation [17] for $t<0$ and $J /|t|=0.3$.

\section{SUMMARY AND DISCUSSIONS}

In this paper, we have studied the single-band $t-J$ model on a triangular lattice, based on variational Monte Carlo (VMC) calculations, with $\mathrm{Na}_{0.35} \mathrm{CoO}_{2} \cdot 1.3 \mathrm{H}_{2} \mathrm{O}$ in mind. The present study, which treats the local correlation precisely, is more reliable than the previous meanfield-type calculations. We have compared the total energy for various pairing symmetries in a small- $J /|t|$ region, changing the doping rate $\delta$ and the sign of $t$. We consider $s$, extended- $s, d$, and $d+i d$ waves for singlet pairing, and $p+i p$ and $f$ waves for triplet pairing. Furthermore, we have studied the stability of Nagaoka ferromagnetism and inhomogeneous states. Main results are summarized as follows.

(1) For $t>0$, the $d+i d$-wave state, which breaks time reversal symmetry, is dominant for $0<\delta \lesssim 0.24(\mathrm{~J} / \mathrm{t}=$ 0.3 ), but the simple $d$-wave state has a quite close energy to that of the $d+i d$ wave for a similar range of $\delta$. The stability of the superconducting state is rapidly lost, as the carrier density goes away from half filling. For $\delta \sim 0$, phase separation may take place. See Fig. 13)(a).

(2) For $t<0$, in a wide parameter range of our concern $(0<\delta \lesssim 0.96,0 \leq J / t \lesssim 0.7)$, the Nagaoka ferromagnetic state defeats the $d+i d$-wave state, which is the most stable among the superconducting states studied. See Fig. [13(b).

(3) Triplet pairing is not realized in the $t$ - $J$ model, although an $f$-wave state becomes slightly more stable than the normal state in the special case of $\delta=0.5$ and $t<0$, where the Fermi surface overlaps the van Hove singularity points.

These results broadly support the previous meanfield-type studies, 14, 15, 16, 17] and is consistent with the high-temperature expansion studies. 21] Now, let us check these results for the triangular $t-J$ model in the light of a model of $\mathrm{Na}_{0.35} \mathrm{CoO}_{2} \cdot 1.3 \mathrm{H}_{2} \mathrm{O}$. Within the $t$ - $J$ model with small $J /|t|$, the range of steady superconductivity is limited to $0<\delta \lesssim 0.24$ with $t>0$, and 

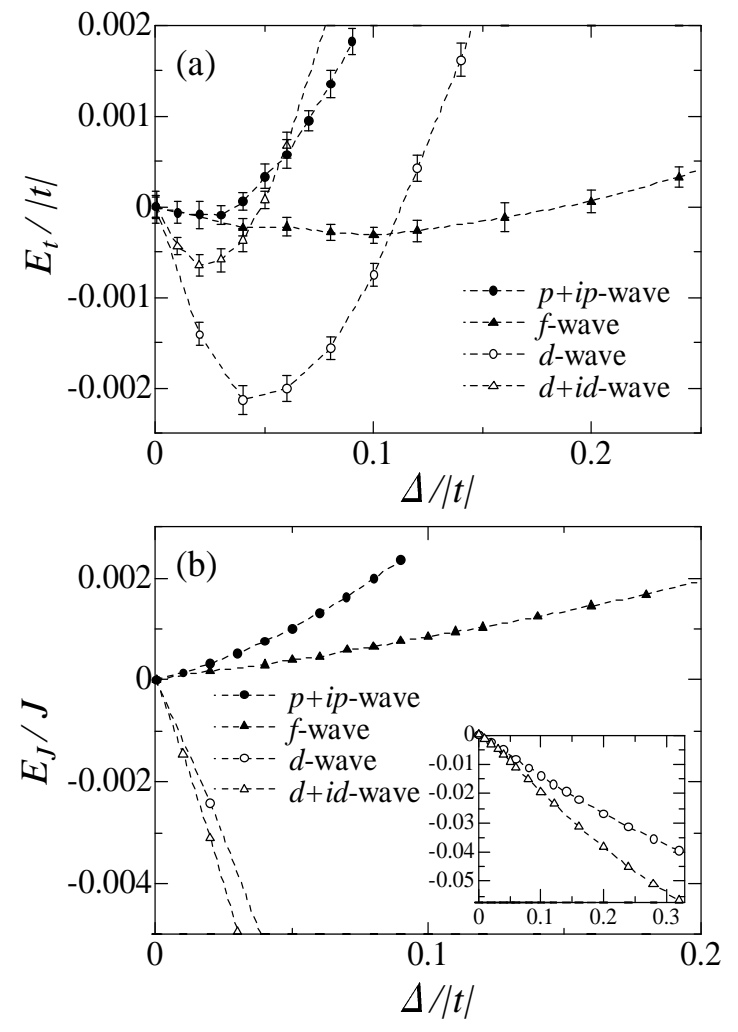

FIG. 11: Comparison of (a) hopping energy and (b) exchange energy among the $p+i p, f, d+i d$ and $d$ waves for $t^{\prime} / t=0.999999(t<0)$ and $\delta=0.5(L=12)$. Here, we assume $J^{\prime} / J=\left(t^{\prime} / t\right)^{2}$. The inset in (b) shows the behavior of the $d$ and $d+i d$ waves in a wider range.

the pairing symmetry is the $d+i d$ (or possibly pure $d$ ) wave. In experiment, although the pairing symmetry of $\mathrm{Na}_{0.35} \mathrm{CoO}_{2} \cdot 1.3 \mathrm{H}_{2} \mathrm{O}$ has not yet established, it is likely that its gap has a line node, 3, [4] and does not break the time-reversal symmetry. [6, 7] Furthermore, the carrier density at which superconductivity appears is limited to a narrow range near $\delta=0.3$. 45] Therefore, it is difficult to conclude that the $t-J$ model successfully describes the properties of $\mathrm{Na}_{0.35} \mathrm{CoO}_{2} \cdot 1.3 \mathrm{H}_{2} \mathrm{O}$ in the present experimental situation. Provided that this discordance originates in the theoretical side, we discuss some related issues briefly in the remainder.

As mentioned in $\S 1$, the results of triplet, especially $f$ wave, pairing were obtained by applying weak-coupling approaches to Hubbard-type models. 11, 12] Among them, Kuroki et al. paid attention to the role of $e_{g}^{\prime}$ band 13., which broadly corresponds to the $t<0$ band. They showed using a FLEX approximation that the $f$ wave state is the most stable. Thus, it is interesting to confirm whether the stabilization of the $f$ wave found for $\delta \sim 0.5$ and $t<0$ in the $t-J$ model is connected to this weak-coupling $f$ wave in the Hubbard model. Actually, the Hubbard-type model became tractable by a VMC method due to a recent improvement of the variational
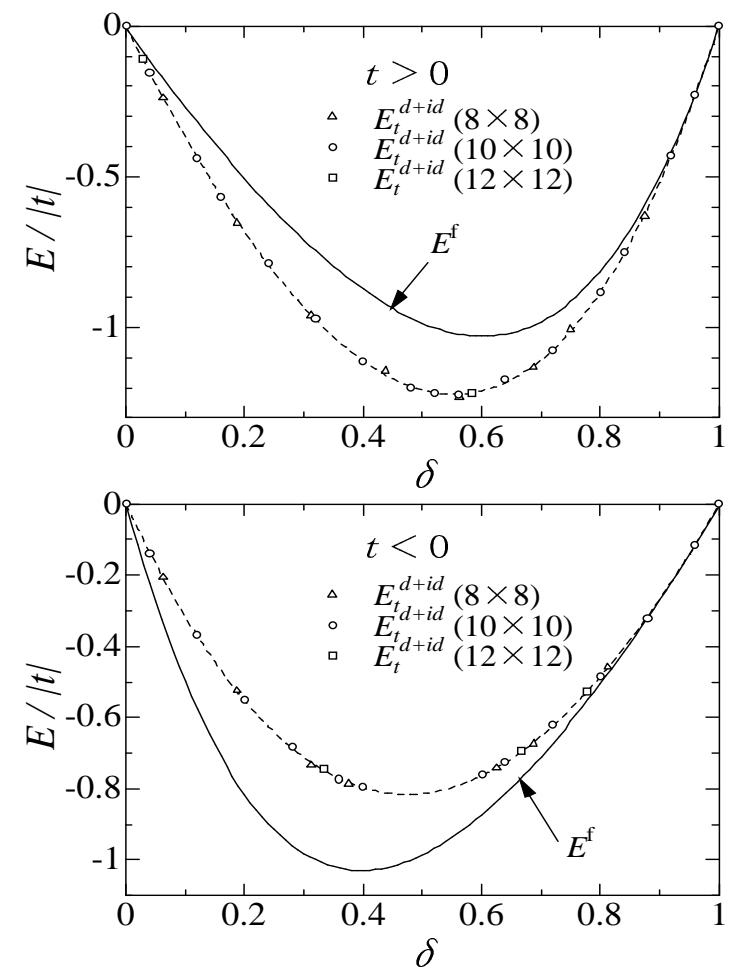

FIG. 12: Comparison of the transfer energy between the optimized VMC values $(J / t=0)$ of the $d+i d$-wave SC state and those of the complete ferromagnetic state $\left(E^{\mathrm{f}}\right)$, estimated analytically through a spinless Fermion model. (a) $t>0$, and (b) $t<0$.

wave functions. 46 .

In this paper, we have used a single-band model as a first step to study the Co-based oxide. However, there exists a possibility that the multiband effect plays an essential role for superconductivity. [8, 10] Actually, recent FLEX and perturbation calculations [9] performed on multi-band Hubbard models yielded results of dominant $f$ and $p$ waves. Here, the $e_{g}^{\prime}$ band again makes a key contribution to superconductivity. To study this effect for large $U / t$ is also an interesting remaining issue.

In the connection of triplet pairing, we finally remark that the pairing symmetry of $\mathrm{Na}_{0.35} \mathrm{CoO}_{2} \cdot 1.3 \mathrm{H}_{2} \mathrm{O}$ can be determined by the phase sensitive experiments like tunneling effect and Josephson effect through the Andreev bound states. 47, 48, 49, 50, 51] In particular, to identify the triplet pairing, it is effective to use the anomalous proximity effect predicted very recently. 52

\section{Acknowledgments}

The authors thank Kazuhiko Kuroki for useful discussions. This work is partly supported by Grant-inAids from the Ministry of Education, Culture, Sports, Science and Technology, by the Supercomputer Center, 

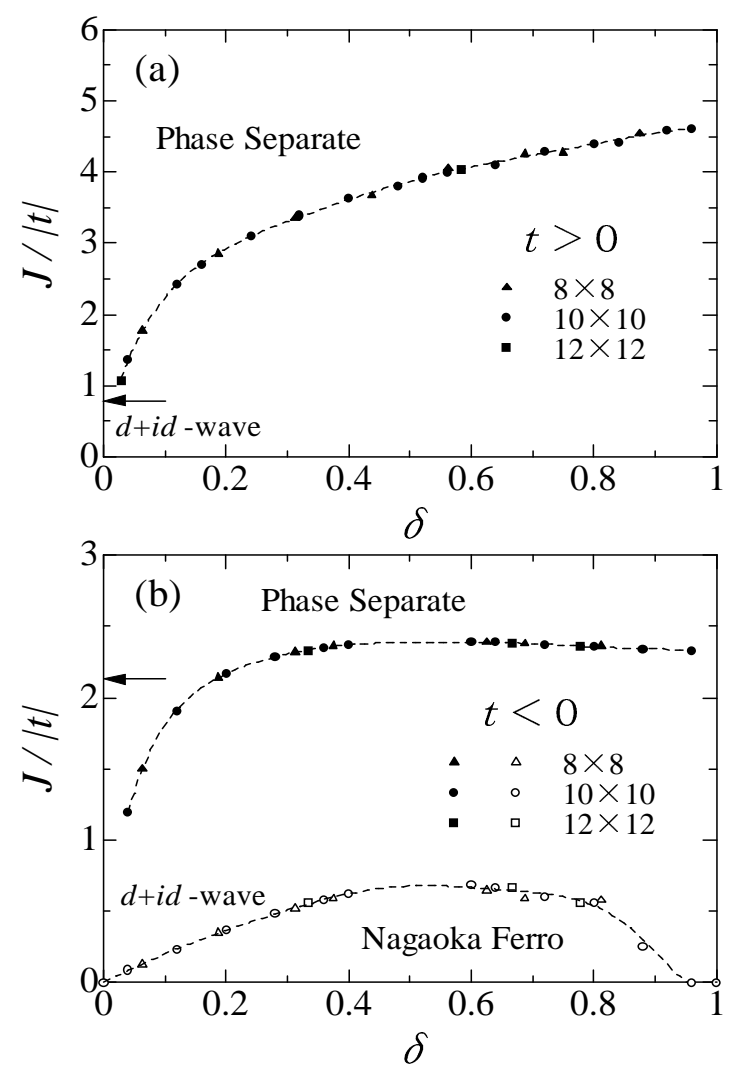

FIG. 13: Phase diagram in the $\delta-J /|t|$ space constructed from the present calculations, for (a) $t>0$ and (b) $t<0$. The arrows on the vertical axis indicate the critical values of the phase separation estimated by the way (ii). Note that the system-size dependence is insignificant.
ISSP, University of Tokyo, and by NAREGI Nanoscience Project, Ministry of Education, Culture, Sports, Science and Technology, Japan, which enables us to carry out the calculations on the computers at the Research Center for Computational Science, Okazaki National Research Institutes.
[1] K. Takada, H. Sakurai, E. Takayama-Muromachi, F. Izumi, R. A. Dilanian and T. Sasaki: Nature 422 (2003) 53.

[2] Y. Kobayashi, M. Yokoi and M. Sato: J. Phys. Soc. Jpn. 72 (2003) 2453.

[3] T. Fujimoto, G. Zheng, Y. Kitaoka, R. L. Meng, J. Cmaidalka and C. W. Chu: Phys. Rev. Lett. 92 (2004) 047004.

[4] K. Ishida, Y. Ihara, Y. Maeno, C. Michioka, M. Kato, K. Toshimura, K. Takada, T. sasaki, H. Sakurai and E. Takayama-Muromachi: J. Phys. Soc. Jpn. 72 (2003) 3041.

[5] T. Waki, C. Machioka, M. Kato, K. Yoshimura, K. Takada, H. Sakurai, E. Takayama-Muromachi and T. Sasaki: cond-mat/0306036 (2003).

[6] W. Higemoto, K. Ohishi, A. Koda, R. Kadono, K. Ishida, K. Takada, H. Sakurai, E. Takayama-Muromachi and T. Sasaki: cond-mat/0310324 (2003).

[7] Y. J. Uemura, P. L. Russo, A. T. Saviti, C. R. Wiebe, G. J. MacDougall, G. M. Luke, M. Mochizuki, Y. Yanase, M. Ogata, M. L. Foo and R. J. Cava: cond-mat/0403031

[8] W. Koshibae and S. Maekawa: Phys. Rev. Lett. 91
(2003) 257003.

[9] K. Mochizuki, Y. Yanase and M. Ogata: cond-mat/0407094 Y. Yanase, K. Mochizuki and M. Ogata: cond-mat/0407563

[10] H. Yata and Y. Ono: unpublished.

[11] H. Ikeda, Y. Nishikawa and K. Yamada: J. Phys. Soc. Jpn. 73 (2004) 17; Y. Nishikawa, H. Ikeda and K. Yamada: J. Phys. Soc. Jpn. 73 (2004) 1127.

[12] Y. Tanaka, M. Ogata and Y. Yanase, J. Phys. Soc. Jpn. 73 (2004) 319.

[13] K. Kuroki, Y. Tanaka and R. Arita: to be published in Phys. Rev. Lett.(cond-mat/0311619), and cond-mat/0407587

[14] B. Kumar and B. S. Shastry, Phys. Rev. B 68 (2003) 104508.

[15] G. Baskaran, Phys. Rev. Lett. 91 (2003) 097003.

[16] Q. -H. Wang, D. -H. Lee, and P. A. Lee, Phys. Rev. B 69 (2004) 092504.

[17] M. Ogata, J. Phys. Soc. Jpn. 72 (2003) 1839.

[18] T. Watanabe, H. Yokoyama, Y. Tanaka, J. Inoue and M. Ogata: to appear in J. Phys. Chem. Solids.

[19] Y. Nagaoka: Phys. Rev. 147 (1966) 392. 
[20] T. Hanisch, G. S. Uhrig and E. Müller-Hartmann: Phys. Rev. B 56 (1997) 13960.

[21] T. Koretsune and M. Ogata: Phys. Rev. Lett. 89 (2002) 116401, and J. Phys. Soc. Jpn. 72 (2003) 2437.

[22] H. Yokoyama and H. Shiba: J. Phys. Soc. Jpn. 56 (1987) 3570; W. O. Putikka, M. U. Luchini and M. Ogata: Phys. Rev. Lett. 69 (1992) 2288.

[23] As usual, we use the notation $e_{g}^{\prime}$ to distinguish it from $e_{g}$.

[24] D. J. Singh, Phys. Rev. B 61 (2000) 13397.

[25] A. Tanaka and X. Hu: Phys. Rev. Lett. 91 (2003) 257006.

[26] W. L. McMillan, Phys. Rev. 138 (1965) A442.

[27] D. Ceperley, G. V. Chesfer and K. H. Kalos: Phys. Rev. B 16 (1977) 3081.

[28] H. Yokoyama and H. Shiba, J. Phys. Soc. Jpn. 56 (1987) 1490.

[29] H. Yokoyama and M. Ogata: J. Phys. Soc. Jpn. 65 (1996) 3615.

[30] A. Paramekanti, M. Randeria and N. Trivedi: Phys. Rev. Lett. 87 (2001) 217002, and Phys. Rev. B 70 (2004) 054504 .

[31] C. Gros, R. Joynt and T. M. Rice: Phys. Rev. B 36 (1987) 381; H. Yokoyama and M. Ogata: Phys. Rev. Lett. 67 (1991) 3610, and Phys. Rev. B 53 (1996) 5758.

[32] H. Yokoyama and H. Shiba: J. Phys. Soc. Jpn. 59 (1990) 3669; H. Yokoyama: Prog. Theor. Phys. 108 (2002) 59.

[33] M. C. Gutzwiller: Phys. Rev. Lett. 10 (1963) 159.

[34] The coefficient of each electron configuration in eq. (3) can be represented in a determinantal form, so that the effective algorithm of ref. [27]) works for such BCS-type functions. See, J. P. Bouchaud, A. Georges and C. Lhuillier: J. Phys. (Paris) 49 (1988) 553.

[35] P. W. Anderson: Science 235 (1987) 1196.

[36] For example, for $\delta=0.22$, the extended- $s$ wave (1) has a minimum at a finite value of $\Delta$ for $J / t>0.5$ for $t>0$, and for $J / t>0.8$ for $t<0$. For the type (2), the energy minimum is at $\Delta=0$ for at least $J / t<1$.0. However, we leave it for a future study whether the extended- $s$ wave becomes stable for larger values of $J /|t|$, as in the square lattice. 29, 37]

[37] E. Dagotto, J. Riera, Y. C. Chen, A. Moreo, A. Nazarenko, F. Alacaraz and F. Orantoni: Phys. Rev. B 49 (1994) 3548.
[38] At half filling, the pure $d$ wave has a lower value of $E_{\text {tot }}$ than the $d+i d$ wave, for finite systems. Checking the system-size dependence ( $L=4,6,12$ and 18), however, we have found the values of the two symmetries seem degenerate in the $L \rightarrow \infty$ limit. In reality, an antiferromagnetism with $120^{\circ}$ structure, which is outside the scope of this study, is realized at $\delta=0$. We believe this Néel structure immediately breaks as carriers are doped, due to the substantial frustration.

[39] D. J. Singh, Phys. Rev. B 68 (2003) 020503.

[40] In this condition, we replace $E(0)$ with $E\left(\Delta \rightarrow 0_{+}\right)$, because the two values do not coincide.

[41] M. Ogata, M. U. Luchini, S. Sorella and F. F. Assaad: Phys. Rev. Lett. 66 (1991) 2388.

[42] V. J. Emery, S. A. Kivelson and H. Q. Lin, Phys. Rev. Lett. 64 (1990) 475; C. S. Hellberg and E. Manousakis, Phys. Rev. Lett. 78 (1997) 4609.

[43] L. Capriotti, A.E. Trumper and S. Sorella, Phys. Rev. Lett. 82 (1999) 3899.

[44] Within the present SC wave functions, the best value of $E_{J} / J(d+i d$ wave, $L=18)$ at half filling is $-1.2369 \pm 0.0004$, which is approximately $5 \%$ higher than a value obtained using a projector Monte Carlo method, $-1.2958 \pm 0.0001$. 43] Thus, $E^{\mathrm{PS}}<E^{d+i d}$ holds for $\delta \rightarrow 0$, because, then, $E_{t}$ vanishes.

[45] R. E. Schaak, T. Klimczuk, M. L. Foo and R. J. Cava, Nature 424 (2003) 527.

[46] H. Yokoyama, Y. Tanaka, M. Ogata and H. Tsuchiura, J. Phys. Soc. Jpn. 73 (2004) 1119.

[47] Y. Asano, Y. Tanaka, K. Kuroki and S. Kashiwaya: to appear in Physica C (2004).

[48] Y. Tanaka and S. Kashiwaya, Phys. Rev. Lett. 74 (1995) 3451.

[49] S. Kashiwaya and Y. Tanaka, Rep. Prog. Phys. 63 (2000) 1641.

[50] Y. Tanaka, Y. V. Nazarov, and S. Kashiwaya: Phys. Rev. Lett. 90 (2003) 167003;

[51] Y. Tanaka, Y. V. Nazarov, A. Golubov and S. Kashiwaya: Phys. Rev. B 69 (2004) 144519.

[52] Y. Tanaka and S. Kashiwaya: to appear in Phys. Rev. $\mathrm{B}(2004)$. 